\title{
Evolutionary Trends in Rigid Body Dynamics
}

\author{
Hooshang Hemami \\ Dept. of Electrical Engineering \\ The Ohio State University \\ Columbus, Ohio 43210
}

July 25,2002

\begin{abstract}
Certain evolutionary trends from analytical methods to a combination of analytical, computational and measurement methods are addressed in this paper. Implications of these trends in modeling, stability, actuation and control of multi rigid body systems are explored here. This approach makes use of recent technical developments in two areas. Electronic hardware is currently available for computation, for sensing, and for combination of the two. Powerful state space methods and large-dimensional system theory have been developed in concept and in software. The availability of modular, efficient and light weight sensors, computer chips and information processors, and the ease of integration of the electrical and mechanical components make the approach presented here more feasible and applicable to a variety of robotic, humanoid, biorobotic and human modeling applications.

The approach is based on simultaneous representation of a rigid body in four state spaces. Intelligent choice of one or more of these models at any time is possible. The underlying intelligence can switch the system from one to the other when the system gets close to that representation's singular points or manifolds. Combinations of two or more such state spaces are possible for having a redundantly formulated system.

The paper presents the approach, the state spaces involved, and the Lyapunov stability of the system for a single rigid body.

Keywords: rigid body dynamics, internal states, peripheral states, embedding, state space, Lyapunov stability, inverse dynamics, computational base, indirect measurements, coordinate transformation
\end{abstract}

\section{Introduction}

Present state space and input-output representations of rigid body systems have singularity points where the Lipschitz condition for 
existence and uniqueness of solutions are violated. is and EulerRodriguez parameter formulations overcome this difficulty by embedding the physical state space in a state space of larger dimension subject to certain holonomic constraints. The latter approach, while elegant in concept and computationally efficient, obscures physical characteristics and attributes of rigid body systems, actuators and sensors. Additionally, it makes problems of stability, control and trajectory design more difficult.

The state spaces explored here are extensions of conventional state spaces, and retain the physical attributes of the system and at the same time eliminate the singularity issues and points. The drawback of this approach is the requirement of more intensive computation and transformations. However, this drawback is, as stated before, ameliorated by the availability of present day inexpensive and powerful computational and programming tools.

Traditionally, rigid body dynamics have been formulated by the Lagrange [1], the Hamilton [2,3] and Newton - Euler equations [4]. Other state spaces have been proposed [5], and applied [6]. The latter state space of Euler or Bryant angles as position variable's and the angular velocity of the body, as expressed in the principal coordinate body system is a mathematically and, at the same time, physically tractable system. Further, this model yields itself to systematic studies of stability by the Lyapunov method and controller design $[7,8]$. The main limitation of the the latter model is that, for each of the rigid bodies, the Lipschitz condition is only valid for the open interval:

$$
-0.5 \pi<\theta_{2}<0.5 \pi \text {. }
$$

However,in most physical system the range of $\theta_{2}$ is

$$
-\pi<\theta_{2}<\pi
$$

More about the singularity of the equations of motion as formulated can be found in [4]. One of the main objectives of this paper is to eliminate this limitation by taking advantage of the currently existing computational tools. The conceptual point of the approach is to embed the rigid body( or rigid body system) in a larger space of multi state spaces as will be shown later.

Historically, the problem of singularity has been addressed by utilizing either the Euler - Rodriguez (ER) parameters [4], Quaternions [1]. This means, instead of the three Euler or Bryant angles, four state variables are introduced that are constrained to lie on unit sphere in three space. The use of ER parameters makes the study of actuation a, stability and control somewhat cumbersome.

A method of dealing with the singularities is to switch between different state spaces. Three such state spaces, referred to as peripheral state spaces, are presented here. By monitoring the condition 
number of matrices that are prone to be singular, one can, at the appropriate time, transform the system to another state space that avoids singular points. An alternative method is to develop an internal state space that does not suffer from singularity points. Standard Bryant ( or Euler) angles and their derivatives with respect to time can be constructed as outputs from the states of the internal system. The starting point of the present development is to introduce a , so to speak, internal and three peripheral state spaces. The details of this representation are worked out for one rigid body here for completeness. Philosophically speaking the rigid body is embbedded in this multi state space. The question of embedding, merits further consideration on its own. Here a system is embedded in a larger dimensional state space in order to extend its range of stability. The issue of embedding a system in a larger state space than its actual dimension has not received as much attention in general as that of reducing the dimension of a larger system to a more theoretically and analytically manageable size. A much studied case of reduction is that of reducing the dimension of a linear time-invariant system to that of its input controllable, and output observable subspace, in order to make the input output studies of the system more tractable. Embedding in a larger state space can be used for a variety of purposes. Kane [9] has used embedding in rigid body systems in order to compute constraint and contact forces. The ER parameters embed the three-dimensional space in a four-dimensional space in order to avoid singularities. Other applications of embedding to control [10] and stability [7] can be cited. An application to postural stability and role of the vestibular sensory system is considered in the paper. This application addresses specifically the human head and torso control and the role of the human vision system in self location, i.e., awareness of one's position and orientation in space. There are two reasons for selecting this application.

1. Natural systems and specifically the human system are among the most advanced systems we know, and understanding their dynamics and mysteries are a significant scientific challenge for the foreseeable future. Therefore, attempts at reasonable models of natural system behavior, even modest ones as considered here are justified.

2. The contrast between natural and man-made systems should contribute to making progress on both sides.

The structure of the paper is as follows. The internal state space is introduced in section 2, and the role of computation and measurement in its formulation is elucidated. Location and attitude estimation from external measurements is discussed in section 3 . The issues of actuation and control are considered in section 4 . Numerical examples are presented in The peripheral state spaces and their role in computation, control and attitude estimation is illustrated in section 5. Applications and Numerical examples are presented in section 6 , 
and discussions and conclusions appear in section 7 .

\section{The Internal state space Model}

\subsection{Rigid Body Rotation}

The starting point of the present development is to introduce the xyz inertial (spatial) coordinate system (ics) which, at the initial time, coincide with the principal coordinate system of the body(bcs): front, left hand, and top. The three Bryant angles are the roll about the front axis, the pitch about the left axis, and the yaw about the top axis. All positive angles are counterclockwise. The sequence of angles in the first state space is roll, pitch, and yaw. The rotational space is considered first, and it is assumed that the torque vector $N$, expressed in the bcs operates on the system. Let $\Omega$ be the angular velocity of the body expressed in bcs, and let $J$ be the moment of inertia matrix expressed in the bcs. The equations of motion [11] are:

$$
J \dot{\Omega}=f(\Omega)+N
$$

where

$$
f(\Omega)=-\breve{\Omega} J \Omega .
$$

We define the internal state space as follows: Let $\Omega$ be measured by a gyro system, to define three velocity state variables of the system. Suppose the three components of $\Omega$ are integrated with respect to time by an online computation unit to define the position (nonphysical) state variables.

$$
\Xi(t)=\Xi(0)+\int_{0}^{t} \Omega d t .
$$

The state space of $\Xi$ and $\Omega$ is the internal state space, and can serve as the main representation of the system as shown in figure 1.

It is shown below that linear state feedback can be used to globally stabilize the rotation of the rigid body in the physical threedimensional space. The disadvantage of the position state $\Xi$ is that it does not correspond to easily observable physical or geometrically defined parameters. Consequently, the Bryant ( or Euler) angles, and their angular velocities should be computable from the states $\Xi$ and $\Omega$ as shown below or physically measured as shown in section 3 .

Traditionally for attitude control and stability, for gravity compensation or for actuation, it is necessary to relate the rigid body attitude to the external ics system via Euler or Bryant angles $\Theta$ and $\dot{\Theta}$. For this purpose, namely, computation of the Bryant angles, one uses the relationship between $\Omega$ and $\dot{\Theta}$, as given in the Appendix:

$$
\dot{\Theta}=B(\Theta) \Omega
$$




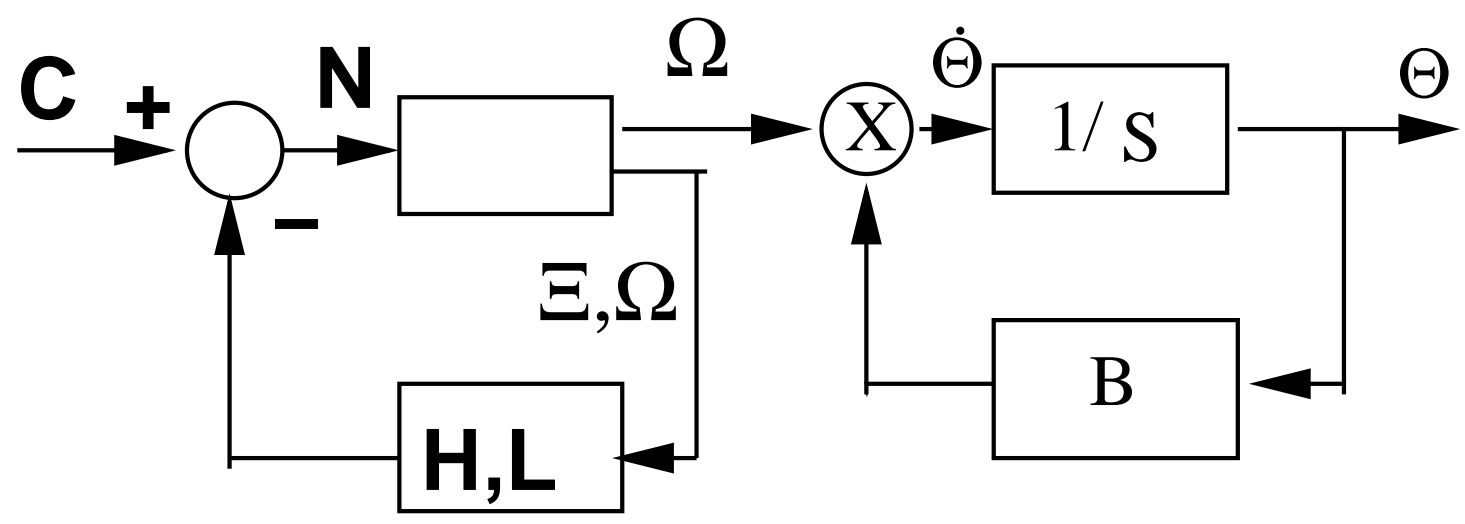

Figure 1: Block diagram of rigid body rotation with internal states and Bryant angle outputs

The needed computation is to first construct the nonlinear matrix $B$, do the above vector multiplication in order to construct $\dot{\Theta}$, and , finally, integrate the result with respect to time in order to arrive at the Bryant angles $\Theta$. This means"

$$
\Theta(t)=\Theta(0)+\int_{0}^{t} B(\Theta) \Omega, d t .
$$

Therefore the Bryant angles and their derivatives are available as outputs of the system. Output feedback can be implemented when the inputs or system parameters depend on the Bryant angles and their derivatives.

The measurement system needed is to sense either the angular velocities $\Omega$, and integrate them once to obtain $\Xi$. Alternatively, angular accelerations can be sensed, and a two step integration provides the velocity and position states.

\section{$2.2 \quad$ Rigid Body Translation}

The states here are the linear velocities and position of the center of gravity of the body. Linear accelerometers, as sensors, with a two step integration provide the internal states.

\subsection{Global Stability}

Consider the rotating system of equation 2 with states $\Xi$ and $\Omega$. Let $H$ and $L$ be two $3 \times 3$ positive definite matrices. The linear state feedback for stability, as shown in figure 1, is taken to be:

$$
N=-H \Xi-L \Omega
$$


Lyapunov methods establishes global stability of the system. Let $v$ be a candidate Lyapunov function that corresponds to the total energy [12] stored in the system:

$$
v_{R}=0.5 \Omega^{\prime} J \Omega+0.5(\Xi) H(\Xi) .
$$

The derivative of $v$ with respect to time is :

$$
\dot{v_{R}}=-\Omega^{\prime} L \Omega .
$$

This derivative is semi-negative definite. Using la Salle's theorem, [13] one can show that the system is globally stable. An analogous linear position and velocity feedback can stabilize the translational motion of the center of gravity.

\section{Location and Attitude Estimation}

Consider a rigid body located in space. Assume there is an inertial coordinate system, and a body coordinate system whose origin is at the center of gravity of the body. Let the coordinates of the center of gravity be $x_{c}$ in the ics. Assume the rigid body is held stationary at an unknown attitude specified by the Bryant angles and $x_{b}$ are, respectively, the known coordinates of the same point on the body on the two coordinate systems. It is known that

$$
x_{i}=x_{c}+A(\Theta) x_{b}
$$

Suppose these coordinates are known at least for three non-coplanar points on the body. These equations can be manipulated in order to suppress $x_{c}$, and sequentially process the resulting equation, to be developed below, to arrive at an estimate of $\Theta$, and hence the attitude(orientation) of the body. These estimates can, subsequently, be used in equation9, to arrive at $x_{c}$, and hence locate the rigid body in the ics. Alternatively, the attitude of the rigid body, namely, specifying the Bryant angles can be derived from on line integration of equation 4 or from measurement of $A$ and a subsequent estimation of the Bryant angles, see for details [14]. A sequential estimation method is discussed here that provides exact angles in the four quadrants as long as equation 1 is satisfied. Alternatively, the measurement technique can be used to calibrate the system and derive the initial values of the Bryant angles at $t=0$.

For simplicity, we assume in the following development that the origins of the ics and bcs coincide. Suppose three non-coplanar points are specified on the rigid body and their Cartesian coordinates in the bcs is known. Let $X_{b}$ be a $3 \times 3$ matrix whose columns are these Cartesian coordinates. By definition, this matrix is nonsingular. Let 
the coordinates of the same three points in the ics system be columns of a matrix $X_{i}$. The measurement techniques and sensors are not further considered in this paper. Two separate cases are presented. In the first case, it is assumed that all components of matrix $X i$ are accurately measured. In the second case, it is assumed that partial knowledge of matrix $X_{i}$ is available in the form of linear projections of matrix $X_{i}$.

\subsection{Total Information}

From the definition of $A$ in the Appendix [4], it follows that, the estimate of $A$ is

$$
A=X_{i} X_{b}^{-1}
$$

Further, from [4], one can estimate $\theta_{1}$ first:

$$
\theta_{1 e}=\operatorname{atan} 2(A(2,3), A(3,3))
$$

This is the first step of the sequential estimation. The second step is to construct $A_{1}\left(\Theta_{1}\right)$, and estimate the product of $A_{2}$ and $A_{3}$ :

$$
A_{2} A_{3}=A_{1}^{-1} X_{i} X_{b}{ }^{-1}
$$

It follows that the estimate of the pitch angle is

$$
\theta_{2 e}=\operatorname{atan} 2\left(A_{2} A_{3}(1,3), A_{2} A_{3}(3,3)\right)
$$

The third step is to construct $A_{2}$, and analogous to step 2, derive $A_{3}$, and from that estimate $\theta_{3 e}$.

Therefore,

$$
A_{3}=A_{2}{ }^{-1} A_{1}{ }^{-1} X_{i} X_{b}{ }^{-1}
$$

It follows that, see Appendix

$$
\theta_{3 e}=\operatorname{atan} 2\left(-A_{3}(1,2), A_{3}(2,2)\right)
$$

This sequential computation, rather than a parallel one, as described by Wittenberg ( [4], page 23), is accurate and unique in all four quadrants of the plane as long as $\theta_{2}$ satisfies the condition of 1 .

The above sequential procedure can be used continuously to estimate the Bryant angles or at the beginning of a task or motion in order to arrive at the initial conditions $\Theta(0)$ needed for integrating equation 4 . The two methods, namely, the integration method and the sequential method can be combined to arrive at a more accurate estimates of the attitude. 


\subsection{Partial Information}

\subsection{1 $\quad x y$ information}

Suppose, as an example, that only the projection of $X_{i}$ in the xy plane of the inertial coordinate system is available. Physically this means all the height components are ignored or lost. Define a projection operator $P$ to be the sum of two single dimensional operators $P_{1}$ and $P_{2}$, see Appendix A. It follows that

$$
P A=P X_{i} X_{b}^{-1}
$$

Following the same sequential approach one can derive estimates for the three Bryant angles:

$$
\theta_{3}=-\operatorname{atan}\left((P A)_{12},(P A)_{11}\right)
$$

The estimate for $\theta_{2}$ is:

$$
\theta_{2}=\operatorname{atan}\left(\left(P A_{1} A_{2}\right)(1,3),\left(P A_{1} A_{2}\right)(1,1)\right)
$$

Finally, $\theta_{1}$ can be derived from:

$$
\theta_{1}=-\operatorname{atan}\left(\left(P A_{1}\right)(2,3),\left(P A_{1}\right)(2,2)\right)
$$

\subsection{2 $\mathrm{xz}$ information}

let $P=P_{1}+P_{3}$. It can be shown that the sequential estimation above can be used to estimate the attitude of the rigid body.

Following the above development, it can be shown that

$$
\theta_{3}=-\operatorname{atan}\left((P A)_{12},(P A)_{1,1}\right)
$$

The estimate for $\theta_{2}$ is:

$$
\theta_{2}=\operatorname{atan}\left(\left(P A_{1} A_{2}\right)(1,3),\left(P A_{1} A_{2}\right)(1,1)\right)
$$

Finally, $\theta_{1}$ can be derived from:

$$
\theta_{1}=\operatorname{atan}\left(\left(P A_{1}\right)(3,2),\left(P A_{1}\right)(3,3)\right)
$$

A third case of partial information is when $P=P_{2}+P_{3}$. This third case does not yield itself to the sequential estimation method. Numerical examples and comparisons between the total information case and the two cases with partial information are given in section 6 .

\section{Peripheral State Feedback}

There are a variety of situations where the dynamics depend on the attitude of the rigid body relative to the inertial coordinate system. Three different cases corresponding to gravity dependence, constrained systems, and actuation are briefly discussed here. 


\subsection{Gravity Dependence}

Suppose the rigid body is connected to the ground by a three degree of freedom, and is actuated by the platform. Let $X$ and $V$ be the translational vectors of position and velocity of the center of gravity of the body in the ics . Let $\Gamma$ be a 3-vector of the forces of contact acting a point with coordinates $R$ in bcs. The vector $G$ is the gravity vector, expressed in ics. The torque $N_{1}$ is the result of all couples and moment of all the remaining forces besides $\Lambda$ operating on the body. The equations of motion of the single rigid body are [15]:

$$
\begin{array}{r}
\dot{\Xi}=\Omega \\
J \dot{\Omega}=f(\Omega)+N_{1}+\breve{\mathcal{R}} A^{\prime} \Lambda \\
\dot{X}=V \\
m \dot{V}=G+\Lambda
\end{array}
$$

Here the Bryant angles, enter the expression for $A(\mid$ Theta $)$ in the equations of motion.

\subsection{Constrained Systems}

Suppose, the rigid body is holonomic ally constrained: the body is permanently connected to an actuated moving base or platform. It is desirable to compute the forces of constraint [16] or eliminate them from the equations of motion.

\subsubsection{Computation of the forces of constraint}

Let the connection, be at the origin of the moving system. Let the motion of the platform origin be a known three-dimensional motion $X_{a}(t)$, described in the inertial coordinate system. The connection to the moving platform can be described by three holonomic constraints:

$$
\begin{aligned}
X+A R & =X_{a}(t) \\
V+A \breve{\Omega} R & =V_{a}(t) . \\
\dot{V}+A(\breve{\Omega})^{2} R-A(\breve{R}) \dot{\Omega} & =\dot{V}_{a}(t)
\end{aligned}
$$

Following similar procedures to those in [16], one can show that the forces of constraint are functions of the internal states, peripheral states, inputs, and gravity. The derivation is straight forward, and is not carried out here.

- subsubsectionElimination of forces 
Often it is desirable to reduce the system dimension from 12 to six. The reduced equations are $[8]$ :

$$
\begin{array}{r}
\dot{\Xi}=\Omega \\
J-b \dot{\Omega}=f_{b}(\Omega)+N_{1}-\breve{\mathcal{R}} A^{\prime}\left(G-m \dot{V}_{a}\right) .
\end{array}
$$

where

$$
\begin{gathered}
\left.J_{b}=J-m(\breve{\mathcal{R}})^{2}\right) . \\
f_{b}=f-\breve{\mathcal{R}} A^{\prime}(G)-m \breve{\mathcal{R}}(\breve{\mathcal{R}} \Omega) \Omega .
\end{gathered}
$$

The reduced system is that of an inverted pendulum fixed at the base, and the Bryant angles appear in the gravity term.

\subsubsection{Measurement of forces}

When the forces of constraint are measurable [?], they may be measured in the bcs or ics. In either case, the Bryant angles enter the equations of motion.

\subsection{Platform Actuation}

Suppose the pendulum is moved by three motors mounted on an ideal gimbal system that connects the rigid body to the platform. Let the motors and the gimbal system be of negligible mass and friction, and have no moments of inertia. The motors produce the couple vector $M$ along the axes of the gimbal system that define the Bryant angle vector $\Theta$. The incremental work, delivered to the body is

$$
d w=M^{\prime} d \Theta .
$$

The instantaneous power $p$, delivered to the pendulum, is

$$
p=M^{\prime} \dot{\Theta}
$$

Let the torque $M_{1}$, expressed in the body coordinate system be $N_{1}$. It follows from equations 24 and 26 that:

$$
N_{1}=B^{\prime} M_{1}
$$

\subsection{Control Implementation}

In many current applications, the desired trajectories are specified in the external coordinate system or the peripheral state spaces. It is assumed here that the controller design follows standard structures $[17,18]$ as shown in figure 2 . 


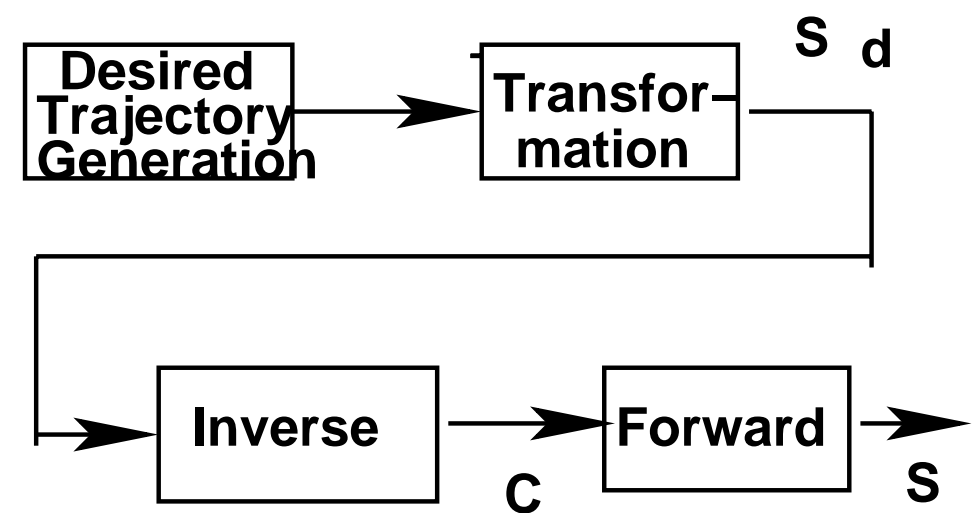

Figure 2: Standard control structure with trajectory construction, transformation of the state when desired, the inverse and the forward systems

For simplicity, the discussion in this section is limited to the rotation subspace of the rigid body. When the internal coordinate system is used, the desired trajectories, expressed in desired $\Theta(t)$ and desired $\operatorname{dot} \Theta(t)$ have to be transformed to the internal coordinate system as shown in figure 3. The latter are input to an inverse system that produces the desired torques $C$. The structure of the inverse system is elaborated here.

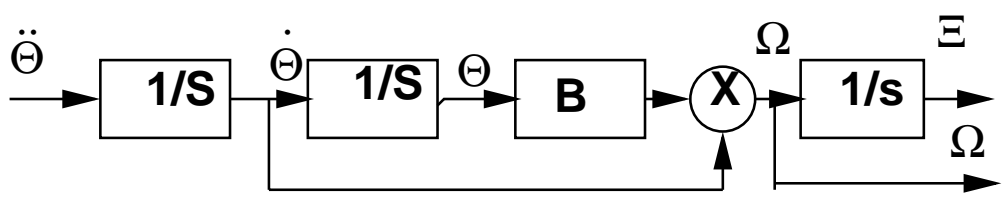

Figure 3: The external desired trajectories are synthesized from integrating the desired accelerations twice, and transforming to internal states $\Xi$ and $\Omega$.

The inverse system has the classical feedback structure as discussed by Zames [19] and Smith [20]. One major advantage of using the internal coordinates is that global stability is guaranteed as will be shown later.

Let the operator $F$ define the forward dynamics of the rigid body rotation as given by equations 2 and 3 , and stabilized by linear internal state feedback $H$ and $L$. Let $\mathbf{C}$ be the input and

$$
S=\left[\Xi^{\prime}, \Omega^{\prime}\right]^{\prime}
$$

the output. The input-output description of the rigid body rotation 
can be operationally written as

$$
S=F(C)
$$

The inverse system can be constructed with the feedback configuration shown in figure 4 where $K$ is a memory-less linear amplifier. It is easy to show that

$$
C=F^{-} 1(S)
$$

We show for an approximate inverse that it is globally stable. Let $K$ be relatively large, such that the term $K^{-1}$ appearing in the dynamics of the inverse can be neglected. Let the input to the inverse system be zero, and let $K$ be structured as two diagonal positive definite $3 \times 3$ matrices:

$$
K=\left[\begin{array}{cc}
K_{1} & 0 \\
0 & K_{2}
\end{array}\right]
$$

The equations of the inverse are given by:

$$
\begin{array}{r}
\Xi(t)=\Xi(0)+\int_{0}^{t} \Omega d t . \\
J \dot{\Omega}=f(\Omega)-H \Xi-L \Omega-K_{1} \Xi-K_{2} \Omega
\end{array}
$$

Following the same development as for the forward dynamics above, one can show that the approximate inverse is globally stable.

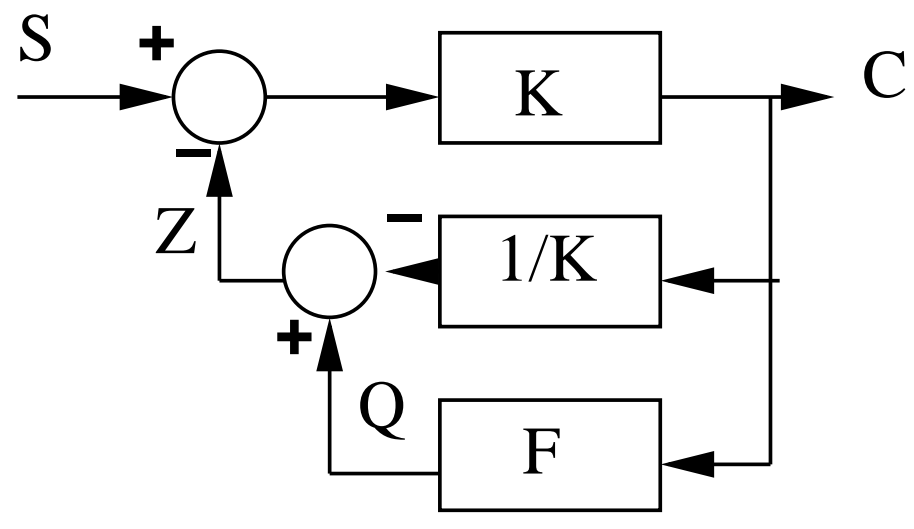

Figure 4: The inverse dynamics system with internal state $S$ and output torque $C$. 


\section{Peripheral State Spaces, and Redundancy}

\subsection{Computations}

In several instances above, namely in computation of the internal state $\Xi$, in measurement of the Bryant angles $\Theta$, and in constructing reference inputs, use is made of matrix $B$. One limitation is that $B$ goes to infinity as $\theta_{2}$ approaches $0.5 \pi$. In order to eliminate this limitation, two additional state spaces are introduced. The three state spaces are, respectively, designated by the sequence of Bryant angles in the definition of the position state: the roll-pitch-yaw as described before, the pitch-yaw-roll state space and the yaw-roll-pitch state spaces. For convenience, these state spaces are abbreviated with an appropriate suffix; r, p, or y. They are discussed further below. These three state spaces are, generically, referred to here as peripheral (or external) state spaces.

\subsection{The Roll state Space}

The $\mathbf{r}$ - state space is given by the states [ $\left.\Theta^{\prime}, \Omega^{\prime}\right]$ ' as defined earlier in the paper and also in the Appendix. The matrices $A_{r}$ and $B i_{r}$ - the inverse of $B_{r}$ - are defined in the Appendix, respectively, as $A$ and $B i$.

\subsubsection{The Pitch State Space}

The $\mathrm{p}$ - state space is characterized by the position state $\Phi$ - a sequence of pitch, yaw and roll, and the angular velocity vector

$$
\Omega_{p}=\left[\omega_{2}, \omega_{3}, \omega_{1}\right]^{\prime} .
$$

The angular velocity $\Omega_{p}$ is related to $\Omega_{r}$ by a $3 \times 3$ permutation transformation $T_{1}$. The matrices $A_{p}$ and $B i_{p}$ are as follows:

$$
A_{p}(\Phi)=A_{1}\left(\phi_{1}\right) A_{2}\left(\phi_{2}\right) A_{3}\left(\phi_{3}\right)
$$

and,

$$
B i_{p}=B i(\Phi)
$$

The relation between $\Theta$ and $\Phi$ is in the following nonlinear implicit equation:

$$
A_{r}(\Theta)=A_{2}\left(\phi_{1}\right) A_{3}\left(\phi_{2}\right) A_{1}\left(\phi_{3}\right)
$$

\subsection{The Yaw - State Space}

The $\mathrm{y}$ - state space is characterized by the position state $\Psi$ - a sequence of yaw, pitch, and roll, and the angular velocity vector

$$
\Omega_{y}=\left[\omega_{3}, \omega_{1}, \omega_{2}\right]^{\prime} .
$$




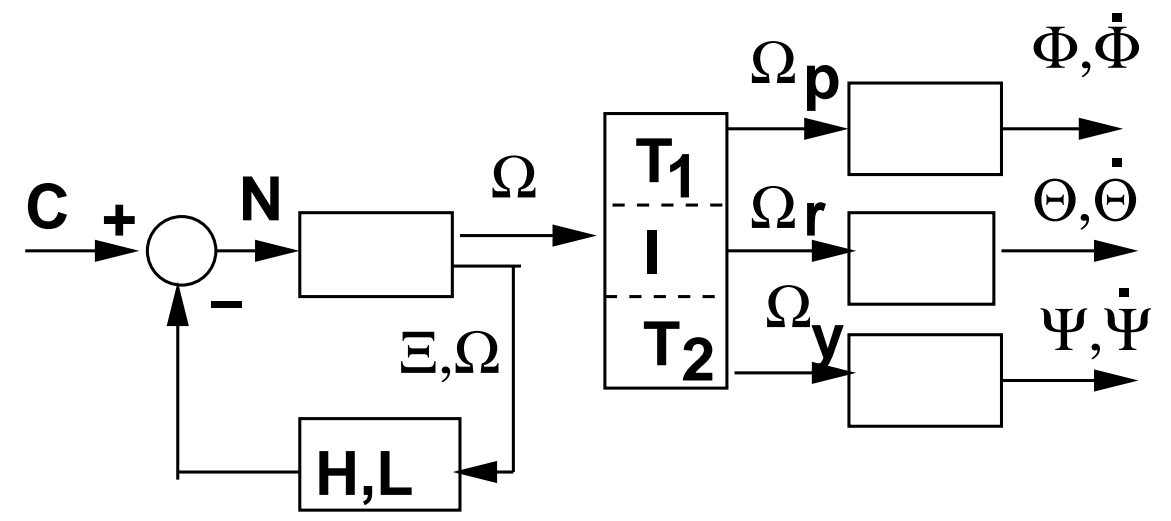

Figure 5: The free rigid body with internal states $\mid X i$ and $\Omega$, globally stabilizing state feedback $H$ and $L$, and three sets of standard output states.

The vector $\Omega-y$ is related to $\Omega_{r}$ by a permutation transformation $T_{2}$ - The matrices $A_{y}$ and $B i_{y}$ are as follows:

$$
A_{y}(\Psi)=A_{1}\left(\psi_{1}\right) A_{2}\left(\psi_{2}\right) A_{3}\left(\psi_{3}\right)
$$

The relation between $\Omega_{y}$ and $\operatorname{dot} \Psi$ is by

$$
B i_{y}(\Psi)=B i(\Psi)
$$

The relationship between $\Theta$ and $\Psi$ in implicit form is

$$
A_{r}(\Theta)=A_{3}\left(\psi_{1}\right) A_{1}\left(\psi_{2}\right) A_{2}\left(\psi_{3}\right)
$$

With the introduction of these three sets of states relating the system to the external world, a measure of redundancy is constructed in the computation and measurements, and one has the ability to switch from one set of outputs to another. The system can be graphically represented as in figure 5 where three transformations $T_{1}$, Identity, and $T_{2}$ are $3 \times 3$ matrices that form a group.

\subsection{Measurements}

The above redundancy is useful when measuring the Bryant angles. When the measured second angle is near \pm 90 degrees, a different external state space may be desirable. For measurement purposes, the state variables as well as the equations of the system have to be transformed. The easiest way to present these transformations is to apply simultaneously the coordinate transformation to both the body 
and the inertial coordinate systems. As discussed earlier, let $T_{1}$ be:

$$
T_{1}=\left[\begin{array}{lll}
0 & 1 & 0 \\
0 & 0 & 1 \\
1 & 0 & 0
\end{array}\right] \text {. }
$$

It follows that

$$
\Omega_{p}=T_{1} \Omega_{r}
$$

The transformation of the position states, namely, the Bryant vector of angles is more involved, since the attitude in ics is the same physical configuration and must be numerically maintained. Suppose the matrices of the coordinates of the fixed point on the body in the inertial and body system are transformed to the $p$ - state space:

$$
X_{i p}=T_{1} X_{i r}
$$

and

$$
X_{b p}=T_{1} X_{b r}
$$

Similarly, let $T_{2}=\left(T_{1}\right)^{-1}$. It follows that the estimate of $A_{p}$

$$
A_{p}=X_{i p}\left(X_{b p}\right)^{-1}=T_{1} X_{i r}\left(X_{b} r\right)^{-1} T_{2}
$$

Alternatively

$$
A_{p}=T_{1} A_{r} T_{2} .
$$

From the latter equation, it follows that the angles $\Phi$ can be estimated by the same sequential computation before.

\subsubsection{Roll Space to Yaw Space Transformation}

This transformation is defined by the permutation of coordinates by $T_{2}$ defined before.

$$
\Omega_{y}=T_{2} \Omega_{r}
$$

The external construction of the Bryant angles with the use of the permutation transformation group defined above is depicted in figure 6.

\section{Application, and Numerical Examples}

\subsection{Application to Human Stability and Self-location}

In this application, first the functional vestibular machinery and their role in postural stability are briefly presented. The second part of the application deals with combining the vestibular and the visual system for self location. 


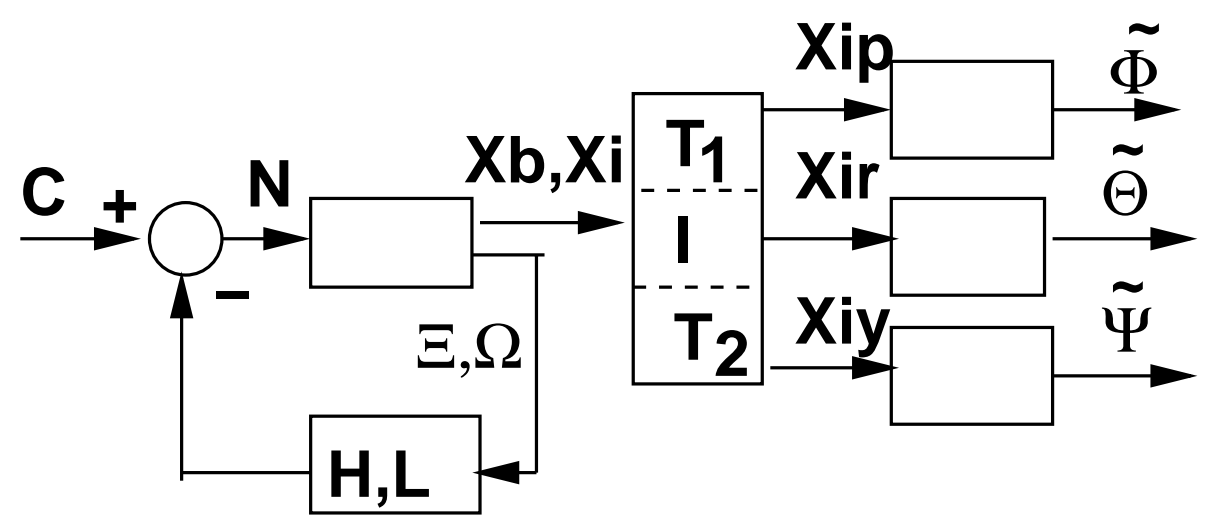

Figure 6: The free rigid body with externally sequentially computed Bryant angles in the three external state spaces

\subsubsection{The vestibular sensory system}

In this section, a functional model of the labyrinth system, and the otolith organs is discussed.

It is generally understood that the labyrinth system senses and measures the angular velocity of the head [21-23]. It is generally agreed that the outputs of these sensors are integrated with respect to time to derive position information in the internal coordinate system $[19,24,25]$. The position information, thus derived is instrumental in the control of the motion of the eyes, i.e., the vestibular Ocular reflex(VOR). These position signals can also be used to stabilize the position of the head relative to the torso and the position of the torso in a sitting position, modeled as an inverted pendulum. For the purpose of elucidation of the above statements, suppose the neck muscles are voluntary made stiff such that the head, the neck and the torso behave as one rigid body. The outputs of the labyrinth system and their integral with respect to time provide position and velocity information in the internal coordinate system. This information, used as position and velocity feedback can stabilize the torso, neck, head system.

Additionally, the output $\Omega$ of the vestibular system can be integrated through the nonlinear mechanism of figure 1 . The latter computation involves construction of matrix $B(\Theta)$. However, as discussed below, it appears that the otolith organs directly provide sine and cosine functions of the yaw and pitch angles of the head. Therefore, it appears that with the availability of such measurements, the task of obtaining the pith and yaw angles is much easier than the nonlinear computational feedback diagram of figure 1. Suppose, for 
completeness, that the central nervous system uses information from the neck muscles to estimate yaw or estimate all the three Bryant angles of the head [26]. Therefore, the natural system appears to use other than the sequential estimation approach, discussed in this paper, to arrive at the orientation of the head.

The role of otolith sensory organs is considered here. The otolith organs measure the linear angular acceleration of the head [27]. The zero frequency component of the sensed acceleration is gravity. This dc measurement can be used by the musculo-skeletal system to compensate for gravity. Wilson and Peterson [21] show responses of the otolith organs to roll and pitch angles of the head(figure 30, page 822). The responses can provide the central nervous system with sine and cosine functions of the roll and pitch angles. These sines and cosines are needed to compensate for gravity torque, and perhaps in construction of $A(\Theta)$ and $B(\Theta)$. The nonzero frequency component, i.e., the ac component of this measurement can be integrated one or twice to provide velocity and position location for the head, and therefore contribute to locating oneself in space. In a sitting position, and when the neck muscles are stiff, the linear position and velocity of the head relative to the chair, can be computationally (or neurally) scaled to derive the linear velocity and position of the center of gravity of the torso.

\subsubsection{Self Location and Orientation}

It was discussed earlier, how a sensor system that measured the coordinates of three non-coplanar points could computationally arrive at the attitude of the body - the orientation. The same information is also a measure of the location of the object in space(ics). In living systems a corresponding sensor system are the eyes, and they are an integral part of the rigid body rather than being installed in the external world.

The issue to be discussed here is how a pair of simple eyes can be used to locate the rigid body in space, or, locate its distance relative to a set of fixed points in space. As was states before, let us assume that the attitude estimation has already been solved by other sensory systems as discussed above.

For this purpose, we assume that the eyes have a simple pin hole geometry and project the external world on an plane of their retinas. further, let us assume that the coordinate system of both eyes and the rigid body have parallel axes. Consider a fixed point $A$ in the inertial coordinate system have coordinates $X_{b}$ in the bcs. There are two point images of point $A$ - one in each eye. These two images on the two retinas are known by their coordinates in the eyes' coordinate system: $X_{r}$ for the right eye, and $X-l$ for the left eye. Let the coordinates systems of the eyes be centered at the pin holes 7 , and 
let the pin holes have coordinates $R_{r}$ and $R_{l}$, respectively, for the right and the left eye.

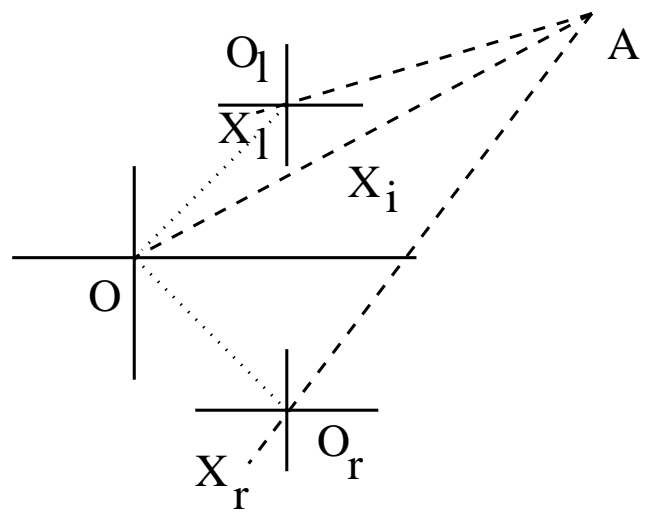

Figure 7: Sketch of the body coordinate system with two eyes in known locations relative to the rigid body, and, modeled as pin hole cameras with images of point A whose coordinates are known in each eye

Let $C_{r}$, and $C_{l}$ be two positive scaler proportionality factors. It can be seen, from figure7 and by geometrical reasoning, that the following two equations correspond to two lines in space. Further, these lines are in the plane formed by the pin holes $O_{r}$ and $O_{l}$ and point A. Therefore, if the lines are not parallel, there is a solution and it is unique.

$$
\begin{array}{r}
X_{i}=R_{r}-C_{r} X_{r} \\
X_{i}=R_{l}-C_{l} X_{l}
\end{array}
$$

The parameters $R_{r}, R_{l}, X_{r}$ and $X_{l}$ are known in these two equations and $X_{i}$ and the proportionality coefficients $C-r$ and $C_{l}$ are the unknowns. The intersection of the two lines is point $A$ and the solution for the unknown parameters afford the coordinate $X-i$ of point $\mathbf{A}$ in the rigid body coordinate system(bcs). Therefore, the location of the rigid is known relative to one fixed point.

To solve for the two unknown parameters, one first equates the two equations:

$$
R_{r}-C_{r} X_{r}=R_{l}-C_{l} X_{l} .
$$

The inner product of the latter by a vector, respectively, orthogonal to $C_{r}$ or $C_{l}$ renders a solution for $C_{l}$ or $C_{r}$.

In the central nervous system, a parallel neural network could simultaneously solve many such equations, and identify the position of the rigid body relative to a larger number of external fixed points. 


\subsection{Numerical Examples}

\subsubsection{Stability of Rotation Motion}

In this example the global Lyapunov stability of the rotational motion of a rigid body is demonstrated. The rigid body parameters are given in Table 1. It is disturbed by a relatively high initial angular velocity. The initial state is taken to be $[0,0,0,20,100,-20]^{\prime}$. The feedback matrices $H$ and $L$ are taken to be diagonal:

$$
H=\operatorname{diag}[400,100,80],
$$

and

$$
L=\operatorname{diag}[20,20,14] \text {. }
$$

Table 1: Numerical values for one rigid body.

\begin{tabular}{|l|c|c|c|}
\hline & symbol & value & unit \\
\hline \hline mass, & $\mathrm{m}$ & 41.00 & $\mathrm{~kg}$ \\
\hline principal moment of inertia & $\mathrm{j} 1$ & 10.0 & $\mathrm{~kg} m^{2}$ \\
\hline principal moment of inertia & $\mathrm{j} 2$ & 8.0 & $\mathrm{~kg} m^{2}$ \\
\hline principal moment of inertia & $\mathrm{j} 3$ & 0.4 & $\mathrm{~kg} m^{2}$ \\
\hline center of gravity & $\mathrm{l}$ & 0.42 & $\mathrm{~m}$ \\
\hline gravity & $\mathrm{g}$ & 10.0 & $\mathrm{~m} / \mathrm{s}^{2}$ \\
\hline
\end{tabular}

Figure 8 shows the trajectories of $\Xi, \Omega$, and $\Theta$ as functions of time. Figure9 shows the Lyapunov function and its derivative with respect to time.

\subsubsection{Attitude Estimation}

In this example three non-coplanar points were selected on the body. The matrix $X_{b}$ for these points is

$$
X_{b}=\operatorname{diag}[10,5,8] .
$$

Ten different attitudes were,randomly, selected for the rigid body. The Bryant angles for these ten attitudes are given in the rows of Table 12 . The Bryant angles are estimated for the attitudes in table 1 from equations 11, 13, and 15. The corresponding estimated angles are given in table 3. As one can see, in cases 4,7 and 10, $\theta_{2}$ exceeds the range given in equation 1 , and the estimated angles for these cases are erroneous. The case of partial information was explored next. The above estimation procedure was repeated for the two cases with partial information as discussed earlier. The results are identical to 

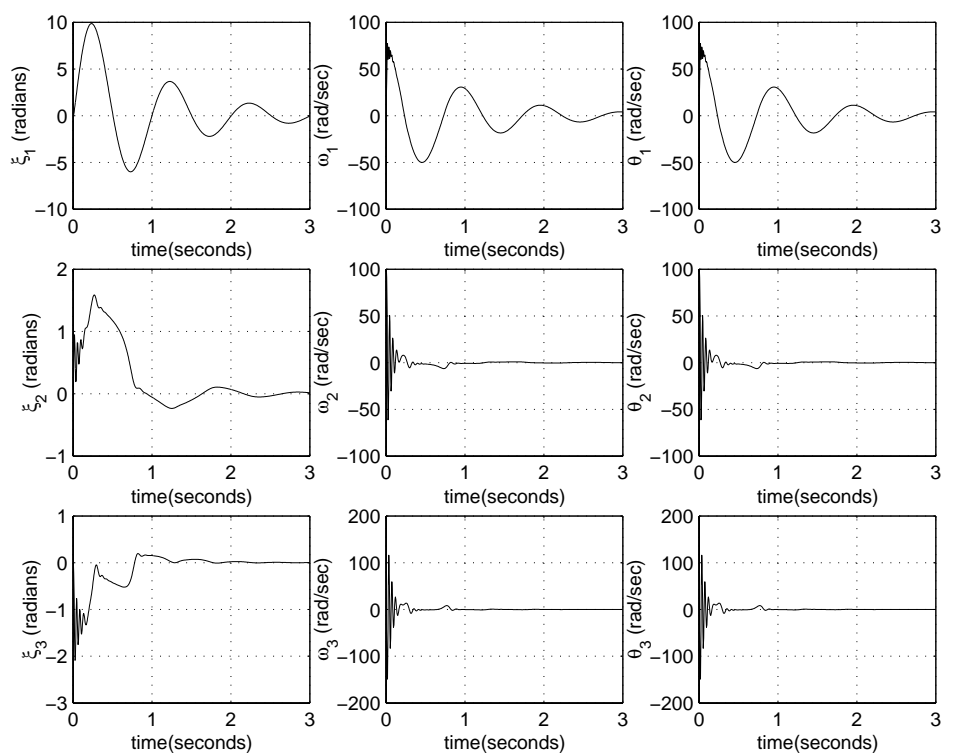

Figure 8: The trajectories of internal states and Bryant angles in the transient simulation of the rigid body with high initial rotational velocities.
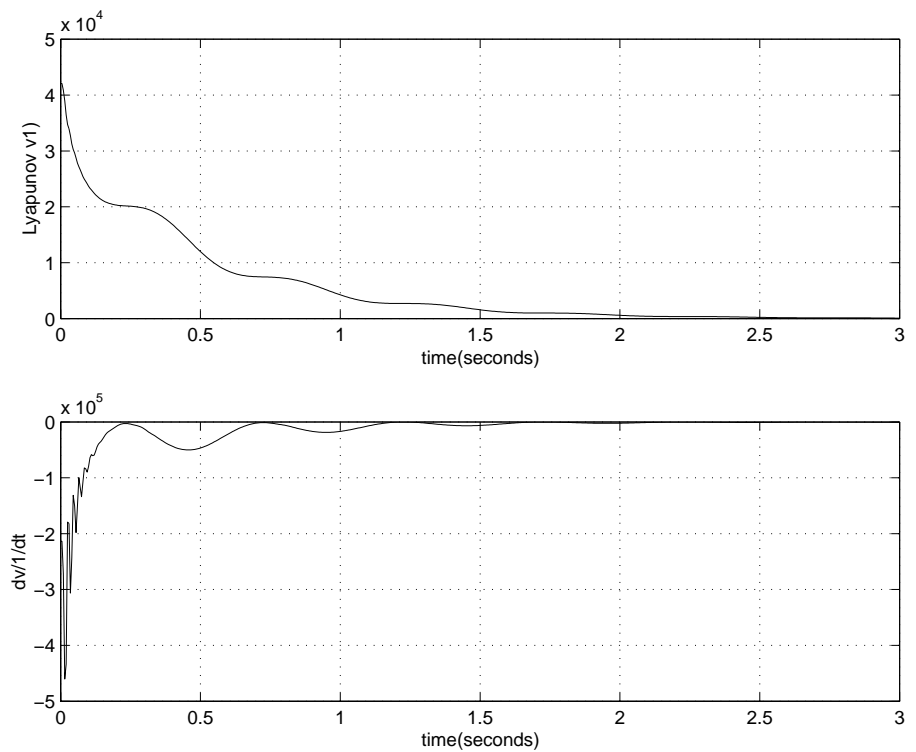

Figure 9: The Lyapunov function and its derivative as functions of time. 
Table 2: Ten Initial sets of Bryant angles for the rigid body attitude.

\begin{tabular}{|l|c|c|c|}
\hline$\theta_{1}$ & $\theta_{2}$ & $\theta_{3}$ & No \\
\hline \hline-3.0441 & 1.5498 & -0.3448 & 1 \\
\hline 2.7118 & -0.2136 & -0.5109 & 2 \\
\hline 2.1743 & 0.1580 & -1.8674 & 3 \\
\hline 1.0810 & 2.1234 & -3.0167 & 4 \\
\hline 1.1384 & -0.7569 & 2.0837 & 5 \\
\hline 0.0177 & 1.3155 & -0.4466 & 6 \\
\hline-1.2270 & -1.9490 & -1.9253 & 7 \\
\hline 1.1444 & -1.2386 & 0.2617 & 8 \\
\hline-2.1925 & 1.2428 & -0.7638 & 9 \\
\hline 2.2609 & 2.2210 & 0.5876 & 10 \\
\hline
\end{tabular}

the ones in Table 3. This confirms the statement that estimation of the Bryant angles is possible with either xy oxz projections, but not with yz projections for the sequence of Bryant angles specified here.

\section{Discussion and Conclusions}

The major tenet of this paper is to discuss certain evolutionary trends in rigid body dynamics by way of introducing a simpler representation of rigid body dynamics. This presentation is more feasible for practice in view of the great progress in computation modules, measurement and sensory devices, information transmission, and integration of the needed subsystems in small packages.

For this purpose, an internal state space and transformations from the internal to standard external state spaces were formulated and presented. Both computation-based and measurement-based transformations were discussed. Specifically, a sequential computational method of estimating the Bryant(Euler) angles was presented that is more general in providing unambiguous answers. Several extensions: gravity dependence, inverse dynamics, and standard control problems were formulated with this framework.

Two measurement systems, one external and mounted on the rigid body, approximately similar to eyes in living systems were introduced and contrasted. One application to natural systems, namely, postural stability of the head and head and torso were discussed. Numerical examples illustrated Lyapunov stability of the system by internal state feedback and global stability, attitude estimation and location with total and partial information.

Philosophically speaking, one may cite advantages for this method. 
Table 3: Estimated Bryant angles for the ten attitudes of the rigid body.

\begin{tabular}{|l|c|c|c|}
\hline$\theta_{1}$ & $\theta_{2}$ & $\theta_{3}$ & No \\
\hline \hline-3.0441 & 1.5498 & -0.3448 & 1 \\
\hline 2.7118 & -0.2136 & -0.5109 & 2 \\
\hline 2.1743 & 0.1580 & -1.8674 & 3 \\
\hline-2.0606 & 1.0182 & 0.1249 & 4 \\
\hline 1.1384 & -0.7569 & 2.0837 & 5 \\
\hline 0.0177 & 1.3155 & -0.4466 & 6 \\
\hline 1.9146 & -1.1926 & 1.2163 & 7 \\
\hline 1.1444 & -1.2386 & 0.2617 & 8 \\
\hline-2.1925 & 1.2428 & -0.7638 & 9 \\
\hline-0.8807 & 0.9206 & -2.5540 & 10 \\
\hline
\end{tabular}

The ideas presented here may be, pedagogically, superior in teaching rigid body dynamics. They yield to more efficient and labor-saving methods of simulation and system integration. Finally, the internal states are candidates to be traced in the central nervous system of natural systems, and, therefore, help to unravel some of the mysteries of the signal processing involved. 


\section{Acknowledgments}

The author gratefully acknowledges the support of the Fundamental Research Laboratories (FRL) of Honda R\&D Americas, Inc, Mountainview,California. The encouragement of Professor Yuan F. Zheng, Chairman of the Department of Electrical Engineering at The Ohio State University is thankfully acknowledged. The editorial assistance of R.L. Rousseau is sincerely appreciated. 


\section{$9 \quad$ Appendix}

Let $\Theta$ and $\Omega$ be, respectively, the Bryant angles and the angular velocity vector of the body expressed in the body coordinate system (bcs). Let $X$ and $V$ be the translational vectors of position and velocity of the center of gravity of the body in the ics system. Let $\Lambda$ be a 3 -vector of force acting a point $C$ on the body whose coordinates are vector $R$ in bcs. In connection with vector $R$, Let the skew symmetric $3 \times 3$ matrix $\breve{\mathcal{R}}[\mathbf{1 5}]$. The vectors of force $G$ and $F$ are, respectively, the gravity vector and the vector of control forces. Let $N$ be the couple of all forces. The equations of motion of the single rigid body are [6]:

$$
\begin{array}{r}
\dot{\Theta}=B(\Theta) \Omega \\
J \dot{\Omega}=f(\Omega)+N+\breve{\mathcal{R}} A^{\prime} \Lambda \\
\dot{X}=V \\
M \dot{V}=G+H+\Lambda
\end{array}
$$

The matrices $A(\Theta), B(\Theta)$ and $R R$ are given below.

Let $A_{1}\left(\theta_{1}\right), A_{2}\left(\theta_{2}\right)$ and $A_{3}\left(\theta_{3}\right)$ be defined by:

$$
\begin{aligned}
& A_{1}\left(\theta_{1}\right)=\left[\begin{array}{ccc}
1 & 0 & 0 \\
0 & \cos \theta_{1} & -\sin \theta_{1} \\
0 & \sin \theta_{1} & \cos \theta_{1}
\end{array}\right] . \\
& A_{2}\left(\theta_{2}\right)=\left[\begin{array}{ccc}
\cos \theta_{2} & 0 & \sin \theta_{2} \\
0 & 1 & 0 \\
-\sin \theta_{2} & 0 & \cos \theta_{2}
\end{array}\right] . \\
& A_{3}\left(\theta_{3}\right)=\left[\begin{array}{ccc}
\cos \theta_{3} & -\sin \theta_{3} & 0 \\
\sin \theta_{3} & \cos \theta_{3} & 0 \\
0 & 0 & 1
\end{array}\right] .
\end{aligned}
$$

Now $A(\Theta)$ can be defined:

$$
A(\Theta)=A_{1}\left(\theta_{1}\right) A_{2}\left(\theta_{2}\right) A_{3}\left(\theta_{3}\right)
$$

The matrix $A$ defines the transformation of a vector in the body coordinate system $X_{b}$ to a vector in the inertial coordinate system $X_{i}$.

$$
X_{i}=A X_{b}
$$

The matrix $B(\Theta)$ is given by: 


$$
B(\Theta)=\left[\begin{array}{ccc}
\frac{\cos \theta_{3}}{\cos \theta_{2}} & \frac{-\sin \theta_{3}}{\cos \theta_{2}} & 0 \\
\sin \theta_{3} & \cos \theta_{3} & 0 \\
\frac{-\sin \theta_{2} \cos \theta_{3}}{\cos \theta_{2}} & \frac{\sin \theta_{2} \sin \theta_{3}}{\cos \theta_{2}} & 1
\end{array}\right] \text {. }
$$

Let vector $R$ have components $r_{1}, r_{2}$, and $r_{3}$. The skew symmetric matrix $\breve{\mathcal{R}}$ is defined as:

$$
\breve{\mathcal{R}}=\left[\begin{array}{ccc}
0 & -r_{3} & r_{2} \\
r_{3} & 0 & -r_{1} \\
-r_{2} & r_{1} & 0
\end{array}\right]
$$

For convenience, the inverse of matrix $B$, designated as $B i$ is also given below:

$$
B i(\Theta)=\left[\begin{array}{ccc}
\cos \theta_{2} \cos \theta_{3} & \sin \theta_{3} & 0 \\
-\sin \theta_{3} \cos \theta_{2} & \cos \theta_{3} & 0 \\
\sin \theta_{2} & 0 & 1
\end{array}\right] .
$$

It is important to relate $B i$ to the three orthogonal transformations $A_{i}, i=1,2,3$. This is easily done with the definition of three projection matrices $P_{1}, P_{2}$ and $P_{3}$, defined below:

$$
\begin{aligned}
& P_{1}=\left[\begin{array}{lll}
1 & 0 & 0 \\
0 & 0 & 0 \\
0 & 0 & 0
\end{array}\right] . \\
& P_{2}=\left[\begin{array}{lll}
0 & 0 & 0 \\
0 & 1 & 0 \\
0 & 0 & 0
\end{array}\right] . \\
& P_{3}=\left[\begin{array}{lll}
0 & 0 & 0 \\
0 & 0 & 0 \\
0 & 0 & 1
\end{array}\right] .
\end{aligned}
$$

With these definitions, the expression for $B i$ is

$$
B i=P_{3}+A_{3}{ }^{\prime} P_{2}+A_{3}{ }^{\prime} A_{2}{ }^{\prime} P_{1}
$$




\section{References}

[1] J. E. Marsden and T. S. Ratiu, Introduction to Mechanics and Symmetry. Springer, 1991.

[2] E. Whittaker, A Treatise on the Analytical Dynamics of Particles and Rigid Bodies. Dover Publications, 1944.

[3] V. Arnold, Mathematical Methods of Classical Mechanics. Springer Verlag, 1989.

[4] J. Wittenberg, Dynamics of Systems of Rigid Bodies. B.G. Teubner, 1977.

[5] A. Isidori, Nonlinear Control Systems. Springer-Verlag, 1989.

[6] H. Hemami, "A state space model for interconnected rigid bodies," IEEE Trans. on Automatic Control, no. 2, pp. 376-382, 1982.

[7] H. Hemami and V. Utkin, "On the dynamics and lyapunov stability of constrained and em bedded rigid bodies," International J. of Control, vol. 75, no. 6, pp. $408-420,2002$.

[8] H. Hemami, "A general framework for rigid body dynamics, stability and control," Journal of Dynamic Systems, Measurement and Control, June 2002.

[9] T. R. Kane and D. A. Levinson, Dynamics, Theory and Applications. McGraw-Hill, 1985.

[10] H. Hemami and B. Wyman, "Modeling and control of constrained dynamic systems with application to biped locomotion in the frontal plane," IEEE Trans. on Automatic Control, no. 4, pp. 526-535, 1979.

[11] A. Shabana, Dynamics of Multibody Systems. Wiley, 1989.

[12] D. E. Koditschek, "The application of total energy as a lyapunov function for mechanical control systems," Contemporary Mathematics, pp. 131-157, 1989.

[13] S. Lefschetz, Stability of Nonlinear Control Systems. Academic Press, 1965.

[14] S. B. Niku, Introduction to Robotics, Analysis, Systems, Applications. Prentice Hall, 2001.

[15] H. Hemami and A. Katbab, "Constrained inverted pendulum for evaluating upright stability," J. Dynamic Systems, Measurement and Control, pp. 343-349, 1982. 
[16] D. Langer, H. Hemami, and D. Brown, "Constraint forces in homonomic mechanical systems," Computer Methods in Applied Mechanics and Engineering, no. 3, pp. 255-274, 1987.

[17] H. Hemami and B. Stokes, "Four neural circuit models and their role in the organization of voluntary movement," Biological $\mathrm{Cy}$ bernetics, no. 2, pp. 69-77, 1983.

[18] L. Jalics, H. Hemami, and B. Clymer, "A control strategy for terrain adaptive bipedal locomotion," Autonomous Robots, vol. 4, pp. 243-257, 1997.

[19] G. Zames, "Nonlinear operators for sysytem analysis," Tech. Rep. 370, Research Laboratory of Electronics, Mass. Inst. of Technology, Cambridge, MA, 02139, August 1960.

[20] O. Smith, Feedback Control Systems. McGraw-Hill, New York, 1958.

[21] V. J. Wilson and W. Peterson, "The role of the vestibular system in posture and movement," in Medical Physiology (V. Mountcastle, ed.), ch. 30, pp. 813 - 836, The C.V. Mosby Co, 1980.

[22] V. Dietz, M. Trippel, and G. Horstmann, "Significance of proprioceptive and vestibulo-spinal reflexes in the co ntrol of stance and gait," in Adaptability of Human Gait, Implicationsfor the Control of Locomot ion (A. E. Patla, ed.), pp. 37-52, North Holland, 1991.

[23] T. Hain, T. Ramaswamy, and M. Hillman, "Anatomy and physiology of the normal vestibular system," in Vestibular Rehabilitation (S. Herdman, ed.), pp. 3-24, Philadelphia: F.A. Davis Company, 2000.

[24] S. Cannon, D. Robinson, and S. Shamma, "A proposed neural network for the integrator of the oculomot or system," Biol. Cybernetics, vol. 49, pp. 127-136, 1983.

[25] G. Gancarz and S. Grossberg, "A neural model of a saccade generator in the reticular forma tion," Neural Networks, vol. 11, pp. 1159-1174, 1998.

[26] J. Kim and H. Hemami, "Coordinated three-dimensional motion of the head and torso by dynamic neural networks," IEEE Transactions on Systems, Man and Cybernetics, part B, no. 5, pp. 653-666, 1998.

[27] K. Barin and J. Durrant, "Applied physiology of the vestibular system," in The Ear: Comprehensive Otology (R. Canalis and P. Lambert, eds.), pp. 113-140, Lippincott Williams and Wilkins, 2000. 